\title{
Performance of Fingerprint Enhancement and Classification using Neural Network
}

\author{
Dr. Heren Chellam G. \\ Assistant Professor of Computer Science, \\ Rani Anna Government College for Women, \\ Tirunelveli-8.
}

\section{ABSTRACT}

Fingerprints are the best and most widely used form of biometric authentication. Everyone is known to have unique, immutable fingerprints. Fingerprints are secure to use because they do not change in one's lifetime. Since fingerprints are unique, even between identical twins, they are perfect for various security uses. The goal of biometric authentication system is to compare two fingerprint images. The authentication in aadhaar card is done by this comparison. Human fingerprints are rich in details called minutiae, which can be used as identification marks for security purposes. Fingerprint authentication systems are based on local ridge features known as minutiae extraction, marking minutiae accurately and rejecting false ones. However, fingerprint images get degraded and corrupted due to variations in skin and impression conditions. To get accurate minutiae, image enhancement techniques are employed prior to minutiae extraction. This paper presents techniquesto verify the identity of fingerprint. So the minutiae points of fingerprint images are classified using support vector machine algorithm for the identification of type of fingerprints.

Keywords: Aadhaar, Authentication, Fingerprint, Minutiae, Support Vector

\section{INTRODUCTION}

Authentication is any process by which you verify that someone is who they claim they are. This usually involves a username and a password, but can include any other method of demonstrating identity, such as a finger print, iris recognition, retina scan or voice recognition. In aadhaar card also authentication is done using fingerprints and iris recognition.

\subsection{Biometric Authentication}

Among all the biometric techniques[1], fingerprint-based identification has been successfully used in numerous applications. Everyone is known to have unique, immutable fingerprints. A fingerprint is made of a series of ridges and furrows on the surface of the finger. The uniqueness of a fingerprint can be determined by the pattern of ridges and furrows as well as the minutiae points[2]. Minutiae points are local ridge characteristics that occur at either a ridge bifurcation or a ridge ending. Fingerprint matching techniques can be placed into two categories minutae-basedand correlation based. Minutiae-based techniques first find minutiae points and then map their relative placement on the finger. However, there are some difficulties when using this approach. It is difficult to extract the minutiae points accurately when the fingerprints oflow quality[3]. Also this method does not take into account the global pattern of ridges and furrows. However, it has some of its own shortcomings. Correlation-based techniques require the precise location of a registration point and are affected by image translation and rotation. 
DOI: https://dx.doi.org/10.26808/rs.ca.i8v1.20

\subsubsection{Fingerprint}

The finger print image acquisition systems are based on the technology such as Frustrated total internal reflection (FTIR), CMOS capacitance, Thermal, Ultrasound. Recently, non-contact fingerprint scanners have been announced that avoid problems related to touch-based sensing methods, including elastic distortion of the skin pattern.

The most commonly used fingerprint features[4] are ridge bifurcations and ridge endings, collectively known as minutiae, which are extracted from the acquired image. The feature extraction process starts by examining the quality of the input gray-level image. Virtually every published method of feature extraction computes the orientation field of the fingerprint image which reflects the local ridge direction at every pixel. The local ridge orientation has been used to tune filter parameters for enhancement and ridge segmentation. From the segmented ridges, a thinned image is computed to locate the minutiae features.

Usually, one has to go through a minutia post-processing stage to clean up several spurious minutiae resulting from either enhancement, ridge segmentation or thinning artifacts. The main goal of the fingerprint authentication module is to report some sort of distance between two fingerprint feature sets accurately and reliably. The authentication function has to compensate for (i) Translation, (ii) Rotation, (iii) Missing features, (iv) Additional features distortion between a pair of feature sets.

Often storage and transmission of fingerprint images involves compression and decompression of the image. The main advantages of fingerprint as a biometric are the high accuracy and low cost of the system.

Due to steady increases in computing power and the advent of unobtrusive, easy-to-use fingerprint sensors, fingerprints are used more frequently as a biometric for identification and recognition[5]. The primary technique for matching newly acquired prints is the extraction and matching of landmarks known as minutiae. Minutiae are areas where the ridges of the print either terminate to form a ridge ending, or split into two new ridges, forming a ridge bifurcation.

Numerous procedures have been proposed for the extraction of minutiae points from fingerprint images[6]. Most of them, however, involve extensive pre-processing of the fingerprint image. A common approach for example, involves the thinning of the ridges, also known as skeletonization or ridge extraction. The process of ridge extraction requires extensive preprocessing, which is time consuming. Each technique improves upon the previous, and the final approach combines the results of the initial three in an attempt to classify the data through clustering.

\subsection{Artificial Neural Network}

Artificial Neural Network's are biologically inspired computer programs to simulate the way in which the human brain process information. It is a very powerful approach for building complex and nonlinear relationship between a set of input and output data. The power of computation comes from connection in a network. Each neuron has weighted inputs, simulation function, transfer function and output. The weighted sum of inputs constitutes the activation function of the neurons. The activation signal is passed through a transfer function which introduces non-linearity and produces the output. During training process, the inter-unitconnections are optimized. Once the network is trained, new unseen input information is entered to the network to calculate the test output. There are many algorithmsavailable in the neural network for classification of data[7,8]. In this paper support vector algorithm is used for finger print images classification. 
DOI: https://dx.doi.org/10.26808/rs.ca.i8v1.20

\section{LITERATURE REVIEW}

\subsection{Authentication via Biometrics}

Authentication is the act of confirming the truth of an attribute of a datum or entity. This might involve confirming the identity of a person or software program, tracing the origins of an artifact, or ensuring that a product is what its packaging and labeling claims to be. The two main directions in the authentication[9] field are positive and negative authentication. Positive authentication is well-established and it is applied by the majority of existing authentication systems[10]. Negative authentication has been invented to reduce cyber attacks. The difference between the two is explained by the following example: Let us assume password-based authentication. In positive authentication, the passwords of all users that are authorized to access a system are stored, usually in a file. Thus the passwords space includes only users passwords andit is usually limited (according to the number of users).

\subsection{Fingerprint Extraction Methods}

Moheb R. et al. [11] proposed an approach to image extraction and accurate skin detection from web pages. This paper proposes a system to extract images from web pages and then detect the skin color regions of these images. As part of the proposed system, using Band Object control, they build a tool bar named "Filter Tool Bar (FTB)" by modifying the PavelZolnikov implementation. In the proposed system, they introduce three new methods for extracting images from the web pages (after loading the web page by using the proposed FTB, before loading the web page physically from the local host, and before loading the web page from any server). These methods overcome the drawback of the regular expressions method for extracting images suggested by IlanAssayag. The second part of the proposed system is concerned with the detection of the skin color regions of the extracted images. So, they studied two famous skin color detection techniques. The first technique is based on the RGB color space and the second technique is based on YUV and YIQ color spaces. They modified the second technique to overcome the failure of detecting complex images background by using the saturation parameter to obtain an accurate skin detection results. The performance evaluation of the efficiency of the proposed system in extracting images before and after loading the web page from local host or any server in terms of the number of extracted images is presented. Finally, the results of comparing the two skin detection techniques in terms of the number of pixels detected are presented.

\subsection{Finger print Verification Method}

Manvjeet Kaur et al. [12] proposed a fingerprint verification system using minutiae extraction technique. Most fingerprint recognition techniques are based on minutiae matching and have been well studied. However, this technology still suffers from problems associated with the handling of poor quality impressions. One problem besetting fingerprint matching is distortion. Distortion changes both geometric position and orientation, and leads to difficulties in establishing a match among multiple impressions acquired from the same finger tip. Marking all the minutiae accurately as well as rejecting false minutiae is another issue still under research. Our work has combined many methods to build a minutia extractor and a minutia matcher. The combination of multiple methods comes from a wide investigation into research papers. Also some novel changes like segmentation using morphological operations, improved thinning, false minutiae removal methods, minutia marking with special considering the triple branch counting, minutia unification by decomposing a branch into three terminations, and matching in the unified $x-y$ coordinate system after a two-step transformation are used in the work. 


\subsection{Segmentation Method}

Ratha et al. [13] proposed an adaptive flow orientation based segmentation or binarization algorithm. In this approach the orientation field is computed to obtain the ridge directions at each point in the image. To segment the ridges, a 16×16 window oriented along the ridge direction is considered around each pixel. The projection sum along the ridge direction is computed. The centers of the ridges appear as peak points in the projection. The ridge skeleton thus obtained is smoothened by morphological operation. Finally minutiae are detected by locating end points and bifurcations in the thinned binary image.

\subsection{Matching Method}

MayankVatsa et al. [14] proposed an combining pores and ridges with minutiae for improved fingerprint verification. This paper presents a fast fingerprint verification algorithm using level-2 minutiae and level-3 pore and ridge features. The proposed algorithm uses a two-stage process to register fingerprint images. In the first stage, Taylor series based image transformation is used to perform coarse registration, while in the second stage; thin plate spline transformation is used for fine registration. A fast feature extraction algorithm is proposed using the Mumford-Shah functional curve evolution to efficiently segment contours and extracts the intricate level-3 pore and ridge features. Further, Delaunay triangulation based fusion algorithm is proposed to combine level-2 and level-3 information that provides structural stability and robustness to small changes caused due to extraneous noise or non-linear deformation during image capture. They defines eight quantitative measures using level-2 and level-3 topological characteristics to form a feature super vector. A 2n-support vector machine performs the final classification of genuine or impostor cases using the feature super vectors. Experimental results and statistical evaluation show that the feature super vector yields discriminatory information and higher accuracy compared to existing recognition and fusion algorithms.

\section{METHODOLOGY}

The uniqueness of a fingerprint can be determined by the pattern of ridges and furrows as well as by features called Minutiae. Minutiae are divide two types such as, Bifurcation and Termination. The Minutiae extraction process includes image enhancement, image segmentation and final Minutiae extraction using the operations such as Ridge Thinning, Minutiae Marking, False Minutiae Removal and Minutiae Representation [15]. Then the extracted minutiae are classified using Support Vector Machine (SVM).

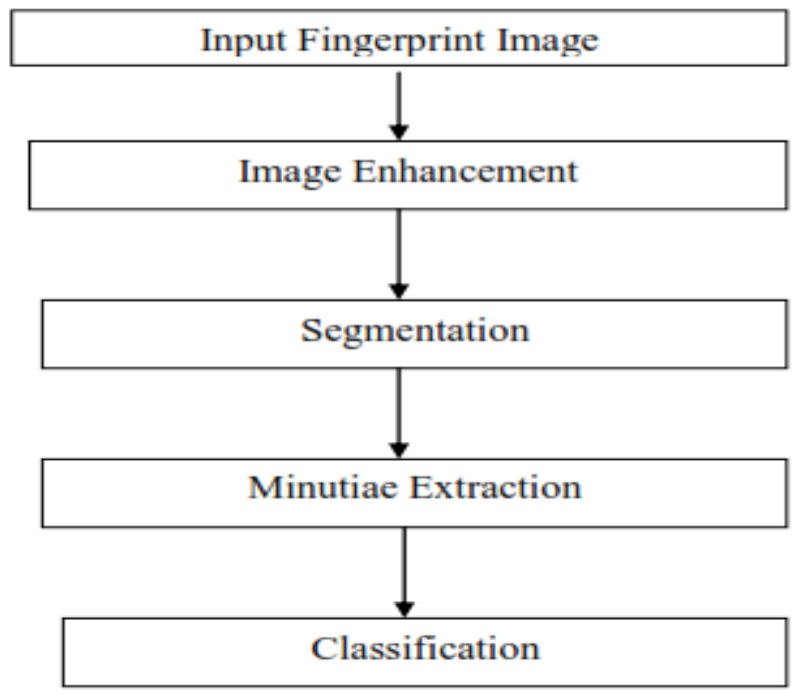

Fig 3.1.Fingerprint Classification Process 
DOI: https://dx.doi.org/10.26808/rs.ca.i8v1.20 International Journal of Computer Application (2250-1797)

Issue 8 Volume 1, January- February 2018

\subsection{Fingerprint Image Enhancement}

The first step in the minutiae extraction stage is Fingerprint Image enhancement. This is mainly done to improve the image quality and to make it clearer for further operations. In this paper we have implemented three techniques, Histogram Equalization, Fast Fourier Transformation and Binarization for image enhancement.

\subsubsection{Histogram Equalization}

Histogram equalization is a technique of improving the global contrast of an image by adjusting the intensity distribution on a histogram. This allows areas of lower local contrast to gain a higher contrast without affecting the global contrast. Histogram equalization accomplishes this by effectively spreading out the most frequent intensity values. The result of the fingerprint histogram equalization is shown in figure 3.2.

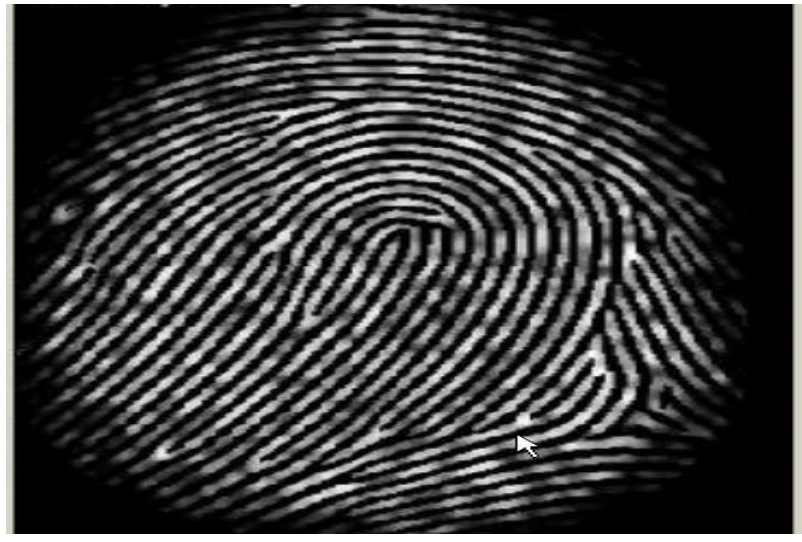

Fig 3.2(a) Original Image

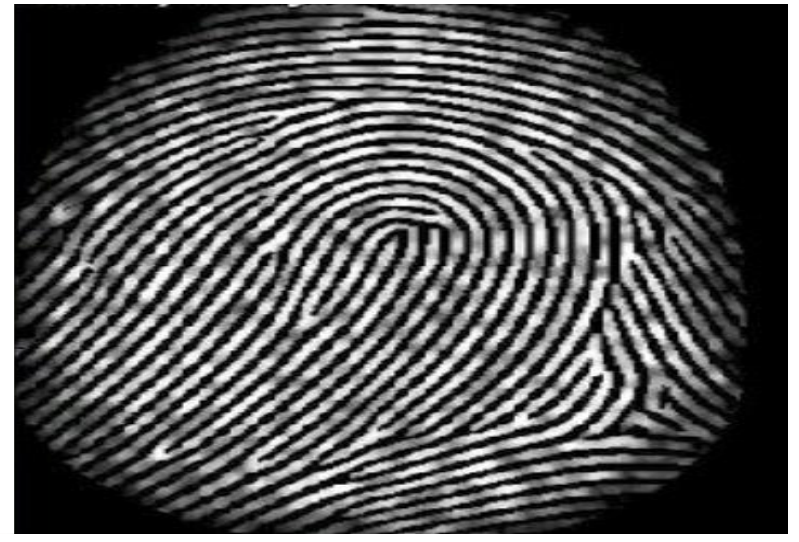

Fig3.2 (b) Enhanced Image after histogram equalization

\subsubsection{Fast Fourier Transformation}

In this method we divide the image into small processing blocks ( 32 x 32 pixels) and perform the Fourier transform according to equation:

$$
F(u, v)=\sum_{x=0}^{M-1 M N-1} f(x, y) \times \exp \left\{-j 2 \pi \times\left(\frac{u x}{M}+\frac{v y}{N}\right)\right\} \quad \text {..Eqn (3.1) }
$$

for $\mathrm{u}=0,1,2, \ldots, 31$ and $\mathrm{v}=0,1,2, \ldots, 31$.

In order to enhance a specific block by its dominant frequencies, we multiply the FFT of the block by its magnitude a set of times. Where the magnitude of the original $F F T=a b s(F(u, v))=\mid F$ $(\mathrm{u}, \mathrm{v}) \mid$.

So we get the enhanced block according to the equation:

$$
g(x, y)=F^{-1}\left\{F(u, v) \times|F(u, v)|^{X}\right\}
$$

where $\mathrm{F}^{-1}(\mathrm{~F}(\mathrm{u}, \mathrm{v}))$ is given by:

$$
f(x, y)=\frac{1}{M N} \sum_{x=0}^{M-1} \sum_{y=0}^{M-1} F(u, v) \times \exp \left\{j 2 \pi \times\left(\frac{u x}{M}+\frac{v y}{N}\right)\right\} . . \text { Eqn (3.3) }
$$

For $\mathrm{x}=0,1,2 \ldots 31$ and $\mathrm{y}=0,1,2 \ldots 31$.

The $\mathrm{k}$ in Eqn (3.2) is an experimentally determined constant, which we choose $\mathrm{k}=0.45$ to calculate. A high value of $\mathrm{k}$ improves the appearance of the ridges by filling up small holes in ridges, but too high value of $\mathrm{k}$ can result in false joining of ridges which might lead to a termination become a bifurcation. Figure 3.3 presents the image after FFT enhancement. 


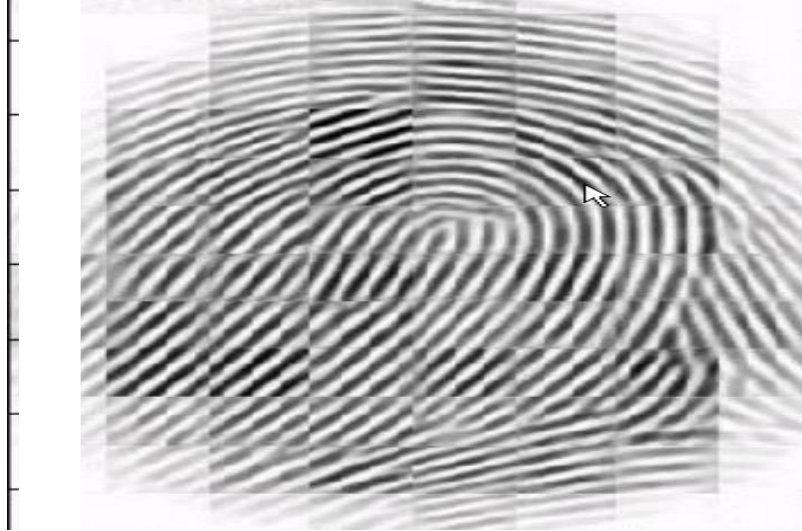

Fig3.3(a) Enhanced Image after FFT

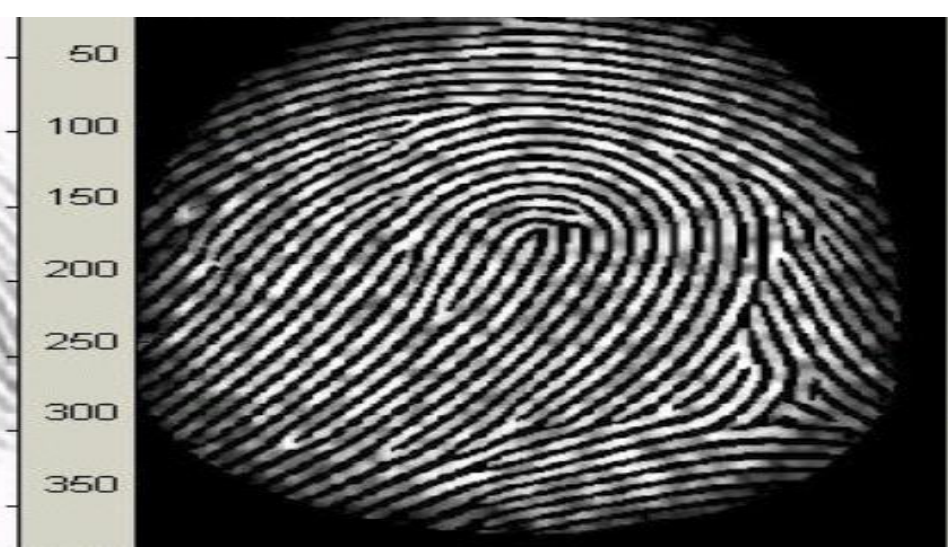

Fig3.3 (b) Image before FFT

The enhanced image after FFT has the improvements as some falsely broken points on ridges get connected and some spurious connections between ridges get removed.

\subsubsection{Image Binarization}

Image Binarization is a process which transforms the 8-bit Gray image to a 1-bit image with 0 -value for ridges and 1-value for furrows. After the operation, ridges in the fingerprint are highlighted with black color while furrows are white.

A locally adaptive binarization method is performed to binarize the fingerprint image. In this method image is divided into blocks of $16 \times 16$ pixels. A pixel value is then set to 1 if its value is larger than the mean intensity value of the current block to which the pixel belongs.

\subsection{Fingerprint Image Segmentation}

After image enhancement the next step is fingerprint image segmentation [16]. In general, only a Region of Interest (ROI) is useful to be recognized for each fingerprint image. The image area without effective ridges and furrows is first discarded since it only holds background information. Then the bound of the remaining effective area is sketched out since the minutiae in the bound region are confusing with those spurious minutiae that are generated when the ridges are out of the sensor.To extract the region of interest, two steps are followed: Block direction estimation and ROI extraction by Morphological methods.

\subsubsection{Block direction estimation}

Here the fingerprint image is divided into blocks of size $16 \times 16$ pixels (W x W) after which the block direction of each block is calculated according to the algorithm:

I. Calculate the gradient values along $\mathrm{x}$-direction $\left(\mathrm{g}_{\mathrm{x}}\right)$ and $\mathrm{y}$-direction $\left(\mathrm{g}_{\mathrm{y}}\right)$ for each pixel of the block. Two Sobel filters are used to fulfill the task.

II. For each block, use following formula to get the Least Square approximation of the block direction.

for all the pixels in each block.

$$
\tan 2 \beta=\frac{2 \sum \sum\left(g_{x} * g_{y}\right)}{\sum \sum\left(g_{x}^{2}-g_{y}^{2}\right)}
$$

The formula is easy to understand by regarding gradient values along $\mathrm{x}$-direction and $\mathrm{y}$ direction as cosine value and sine value. So the tangent value of the block direction is estimated nearly the same as the way illustrated by the following formula.

$$
\tan 2 \theta=\frac{2 \sin \theta \cos \theta}{\cos ^{2} \theta-\sin ^{2} \theta} . \text { Eqn (3.5) }
$$


After finished with the estimation of each block direction, those blocks without significant information on ridges and furrows are discarded based on the following formulas:

$$
E=\frac{2 \sum \Sigma\left(\mathrm{g}_{\mathrm{x}} * \mathrm{~g}_{\mathrm{y}}\right)+\Sigma \Sigma\left(\mathrm{g}_{\mathrm{x}}{ }^{2}-\mathrm{g}_{\mathrm{y}}{ }^{2}\right)}{\mathrm{W} * \mathrm{~W} * \Sigma \Sigma\left(\mathrm{g}_{\mathrm{x}}^{2}+\mathrm{g}_{\mathrm{y}}{ }^{2}\right)} . . \text { Eqn (3.6) }
$$

For each block, if its certainty level $\mathrm{E}$ is below a threshold, then the block is regarded as a background block.The direction map is shown in the following diagram.

\subsubsection{ROI Extraction by Morphological operations}

ROI (Region of Interest) extraction is done using two Morphological operations called open and close. The open operation can expand images and remove peaks introduced by background noise (Fig 3.6). The 'close' operation can shrink images and eliminate small cavities (Fig 3.7).

Fig 3.7 shows the final ROI of the fingerprint which is the bound area after subtraction of the closed area from the opened area. Then the leftmost, rightmost, uppermost and bottommost blocks out of the bound area are discarded.

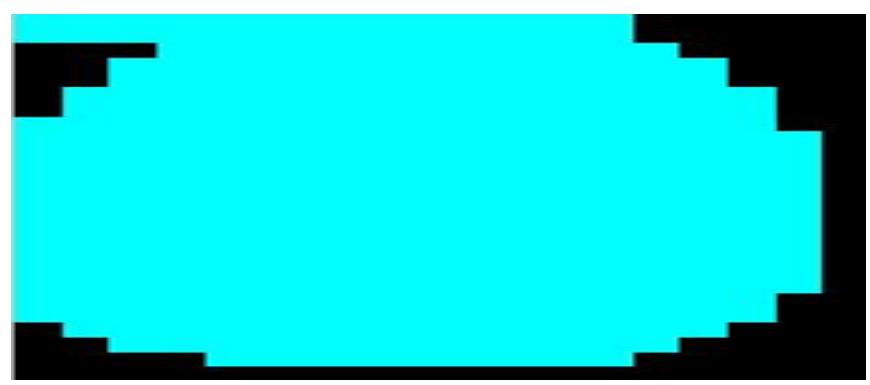

Fig 3.4Originalimagearea

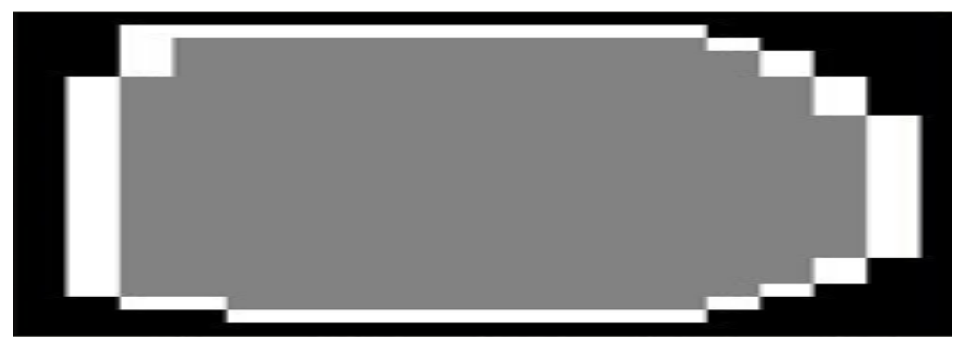

Fig 3.5 Final ROI

\subsection{Minutiae Extraction}

The minutiae[17]from the thinning images are extracted, obtaining accordingly the fingerprint biometric pattern. This process involves the determination of i) whether a pixel, belongs to a ridge or not and, ii) if so, whether it is a bifurcation, a beginning or an ending point, obtaining thus a group of candidate minutiae. Next, all points at the border of the interest region are removed. Then since the minutiae density per unit area cannot exceed this value are substituted by a single minutia located at the center of the cluster. Once, the minutiae extraction[18]process is concluded, the resulting biometric pattern contains typ., Type 1.Arch,2.Left loop 3.Right loop, 4.Whorl, 5.Tented arch.

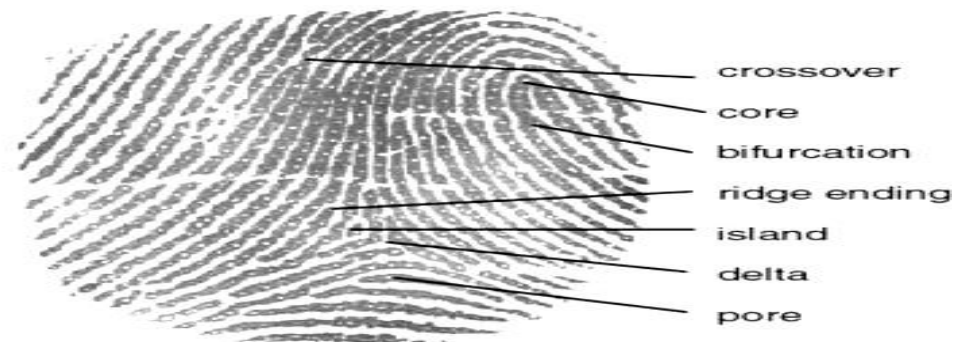

Fig 3.6.Fingerprint image with minutiae details 
Now that we have enhanced the image and segmented the required area, the job of minutiae extraction closes down to four operations: Ridge Thinning, Minutiae Marking, False Minutiae Removal and Minutiae Representation.

\subsubsection{Ridge Thinning}

In this process we eliminate the redundant pixels of ridges till the ridges are just one pixel wide. This is done using the morphological thinning function. The thinned image is then filtered, again using three morphological functions to remove some $\mathrm{H}$ breaks, isolated points and spikes.

\subsubsection{Minutiae Marking}

A minutia marking is now done using templates for each $3 \times 3$ pixel window as follows. If the central pixel is 1 and has exactly 3 one-value neighbors, then the central pixel is a ridge branch (Fig 3.8).If the central pixel is 1 and has only 1 one-value neighbor, then the central pixel is a ridge ending (Fig 3.9).There is one case where a general branch may be triple counted (Fig 3.12). Suppose both the uppermost pixel with value 1 and the rightmost pixel with value 1 have another neighbor outside the $3 \times 3$ window due to some left over spikes, so the two pixels will be marked as branches too, but actually only one branch is located in the small region.

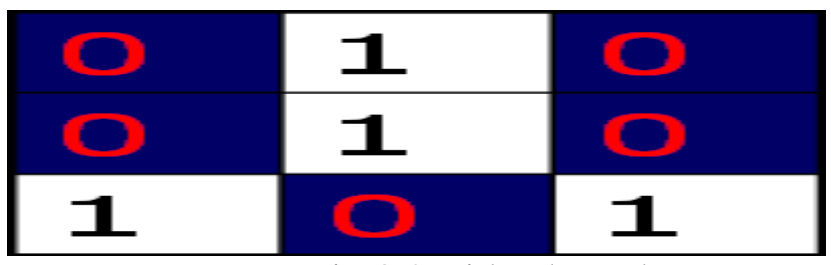

Fig 3.8 Ridge branch

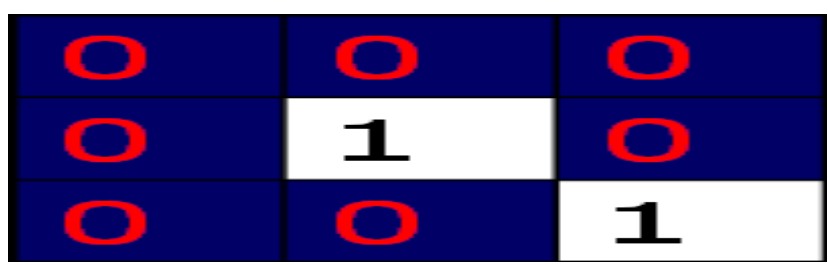

Fig3.9 Ridge ending

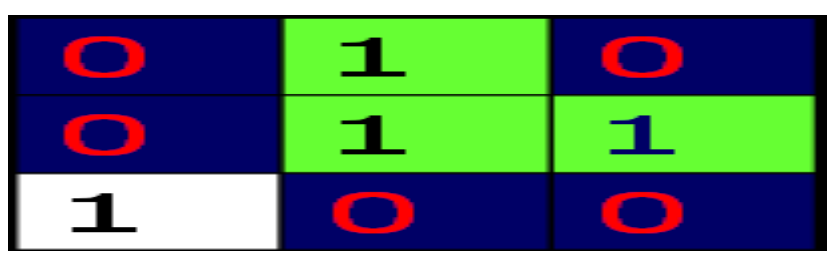

Fig 3.10 Triple count

\subsubsection{False Minutiae Removal}

At this stage false ridge breaks due to insufficient amount of ink \& ridge cross connections due to over inking are not totally eliminated. Also some of the earlier methods introduce some spurious minutia points in the image. So to keep the recognition system consistent these false minutiae need to be removed.

Here we first calculate the inter ridge distance $\mathrm{D}$ which is the average distance between two neighboring ridges. For this scan each row to calculate the inter ridge distance using the formula:

$$
\text { Inter ridge distance }=\frac{\text { sum all pixels with value } 1}{\text { row lengt } h} \text {..Eqn (3.7) }
$$


Finally an averaged value over all rows gives D. All we label all thinned ridges in the fingerprint image with a unique ID for further morphological operations.

Now the following 7 types of false minutia points are removed using these steps (Fig 3.11).

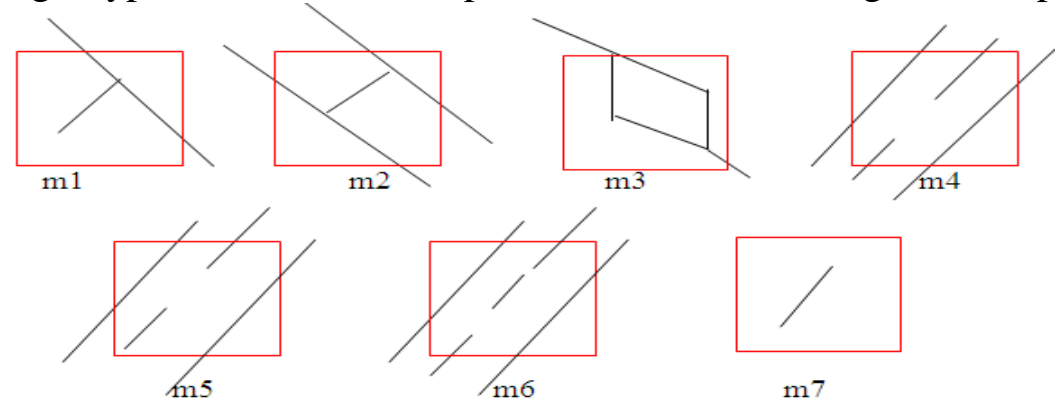

Fig 3.11 False minutia points are removed

- If $\mathrm{d}$ (bifurcation, termination) $<\mathrm{D} \&$ the 2 minutia are in the same ridge then remove both of them (case $\mathrm{m} 1$ )

- If $\mathrm{d}$ (bifurcation, bifurcation) $<\mathrm{D} \&$ the 2 minutia are in the same ridge them remove both of them (case $\mathrm{m} 2, \mathrm{~m} 3$ )

- If $\mathrm{d}$ (termination, termination $) \approx \mathrm{D} \&$ the their directions are coincident with a small angle variation \& no any other termination is located between the two terminations then remove both of them (case $\mathrm{m} 4, \mathrm{~m} 5, \mathrm{~m} 6)$

- If $\mathrm{d}$ (termination, termination) $<\mathrm{D} \&$ the 2 minutia are in the same ridge then remove both of them (case $\mathrm{m} 7)$

where $\mathrm{d}(\mathrm{X}, \mathrm{Y})$ is the distance between 2 minutia points.

\subsubsection{Minutiae Representation}

Finally after extracting valid minutia points from the fingerprint they need to be stored in some form of representation common for both ridge ending and bifurcation[19]. So each minutia is completely characterized by the following parameters 1) x-coordinate, 2) y-coordinate, 3) orientation and 4) ridge associated with it (Fig 3.12)

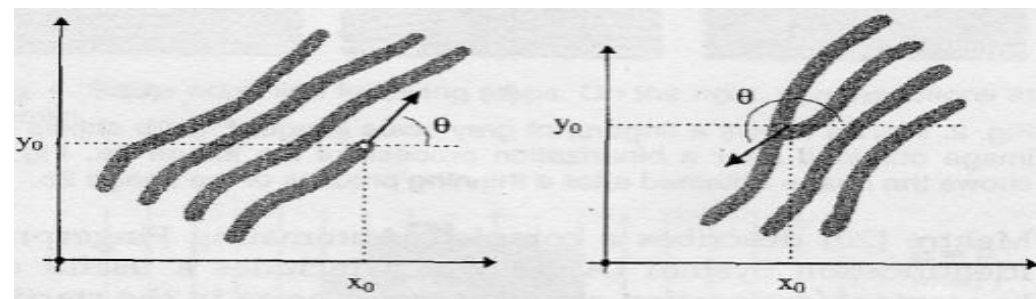

Fig 3.12 Bifurcations and Termination

Actually a bifurcation can be broken down to three terminations each having their own $x-y$ coordinates (pixel adjacent to the bifurcating pixel), orientation and an associated ridge.The orientation of each termination (tx, ty) is estimated by following method. Track a ridge segment whose starting point is the termination and length is D. Sum up all $\mathrm{x}$-coordinates of points in the ridge segment. Divide above summation with D to get sx. Then get sy using the same way.

Get the direction from: $\tan ^{-1} \frac{\mathrm{sy}-\mathrm{ty}}{\mathrm{sx}-\mathrm{tx}}$

\subsection{Support Vector Machine}

A model of SVM is to be designed and trained to recognize the finger code of the databases that are actually used. An imaging system converts each fingerprint image into finger 
code or minutiae matrix code. The result is that each fingerprint image is represented as a vector of 256 real values. Support vector machine when your data has exactly two classes. An SVM classifies data by finding the best hyper plane that separates all data points of one class from those of the other class. The best hyper plane for an SVM means the one with the largest margin between the two classes. Margin means the maximal width of the slab parallel to the hyper plane that has no interior data points. The support vectors are the data points that are closest to the separating hyper plane; these points are on the boundary of the slab, with + indicating data points of type 1 , and indicating data points of type -1 .

The problem, thus, breaks down to converting an N-class problem to be implemented with the help of binary classifiers. The chosen architecture should not only minimize the number of SVMs to be trained, but should also reduce the number of SVMs against which the test pattern has to be tested, thereby reducing both training and testing time. We thus propose a hybrid algorithm with nearest neighbor acting as the first stage and SVMs acting as the second stage.

\section{Experimental Results}

A fingerprint database from the NIST-4 (National Institute of Standards and Technology Special Database 4) is used to test the program's performance[20]. The enhanced fingerprint image is binarized. After the operation, ridges in the fingerprint are highlighted with black color while furrow are white. Ridge thinning is to eliminate the redundant pixels of ridges till the ridges are justone pixel wide. The thinned ridge, map by the filter "minutiae". "Minutiae" compute the number of one-value of each $3 \times 3$ window: If the central is 1 and has only 1 one-value neighbor, then the central pixel is a termination. If the central is 1 and has 3 one-value neighbors, then the central pixel is a bifurcation. If the central is 1 and has 2 one-value neighbors, then the central pixel is a usual pixel.

We have to determine a ROI. For that, we consider the binary image, and we apply an closing on this image and an erosion. With the GUI, I allow the use of ROI tools of MATLAB, to define manually the ROI (Fig 4.5). Once we defined the Region of interest, apply suppress minutiae external to this Region of Interest(ROI) (Fig 4.6). Once determined the different minutiae, we have to find the orientation of each one. To find the orientation of the termination. we analyze the position of the pixel on the boundary of a $5 \times 5$ bounding box of the termination orientation. We compare this position to the Table variable. The Table variable gives the angle in radian. For each bifurcation orientation, we have three lines. So we operate the same process than in termination case three times. Termination and Bifurcation orientation of finger print image is shown in Fig4.7.

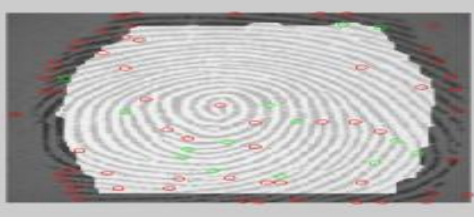

Fig 4.5 Region of Interest

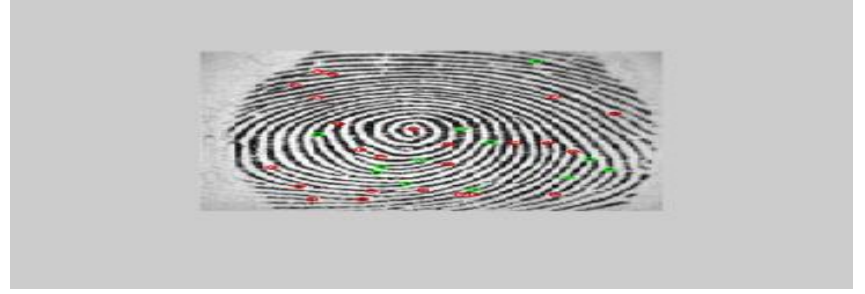

Fig 4.6 Suppress extreme minutiae 


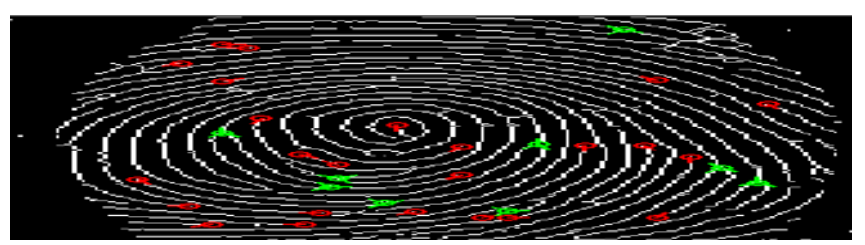

Fig 4.7 Termination and Bifurcation orientation

The finger print classifications[21,22] are considered as arch, left loop, right loop, whorl and tented arch. Support Vector Machine (SVM) algorithm has been applied for the classification of finger print. In this paper 30 dataset are used to train the algorithm. In this algorithm atwostepprocedure is used for fingerprint image classification. The first step computed the singularity points on the fingerprint image based on the maximum variation of its local orientation and the second step classified the fingerprint based on the location of the detected core and delta points. Finally, classified the fingerprints into five groups, such as, whorl, right loop, left loop, arch, and tented arch. The experimental result of finger print image bifurcation, termination and types are shown in Table1.

Table: 1 classification of Finger print images

\begin{tabular}{|l|c|c|c|c|c|c|c|c|c|}
\hline $\begin{array}{l}\text { S.N } \\
\text { o }\end{array}$ & $\begin{array}{c}\text { Patte } \\
\text { rn }\end{array}$ & $\begin{array}{c}\text { No. of } \\
\text { Bifurcati } \\
\text { on }\end{array}$ & $\begin{array}{c}\text { No. of } \\
\text { Terminati } \\
\text { on }\end{array}$ & $\begin{array}{c}\text { Typ } \\
\mathbf{e}\end{array}$ & $\begin{array}{c}\text { S.N } \\
\text { o }\end{array}$ & $\begin{array}{c}\text { Patte } \\
\text { rn }\end{array}$ & $\begin{array}{c}\text { No. of } \\
\text { Bifurcati } \\
\text { on }\end{array}$ & $\begin{array}{c}\text { No. of } \\
\text { Terminati } \\
\text { on }\end{array}$ & $\begin{array}{c}\text { Typ } \\
\text { e }\end{array}$ \\
\hline 1. & $\mathrm{p} 1$ & 9 & 2 & 2 & 16. & $\mathrm{p} 16$ & 33 & 15 & 1 \\
\hline 2. & $\mathrm{p} 2$ & 52 & 22 & 1 & 17. & $\mathrm{p} 17$ & 70 & 7 & 4 \\
\hline 3. & $\mathrm{p} 3$ & 110 & 1 & 4 & 18. & $\mathrm{p} 18$ & 66 & 12 & 2 \\
\hline 4. & $\mathrm{p} 4$ & 123 & 10 & 3 & 19. & $\mathrm{p} 19$ & 53 & 0 & 4 \\
\hline 5. & $\mathrm{p} 5$ & 110 & 1 & 2 & 20. & $\mathrm{p} 20$ & 106 & 6 & 1 \\
\hline 6. & $\mathrm{p} 6$ & 4 & 0 & 2 & 21. & $\mathrm{p} 21$ & 99 & 4 & 1 \\
\hline 7. & $\mathrm{p} 7$ & 22 & 0 & 5 & 22. & $\mathrm{p} 22$ & 79 & 2 & 4 \\
\hline 8. & $\mathrm{p} 8$ & 59 & 6 & 3 & 23. & $\mathrm{p} 23$ & 103 & 5 & 4 \\
\hline 9. & $\mathrm{p} 9$ & 108 & 2 & 4 & 24. & $\mathrm{p} 24$ & 81 & 0 & 1 \\
\hline 10. & $\mathrm{P} 10$ & 83 & 14 & 4 & 25. & $\mathrm{p} 25$ & 84 & 6 & 1 \\
\hline 11. & P11 & 102 & 1 & 1 & 26. & $\mathrm{p} 26$ & 58 & 45 & 2 \\
\hline 12. & P12 & 67 & 14 & 4 & 27. & $\mathrm{p} 27$ & 71 & 0 & 2 \\
\hline 13. & P13 & 107 & 7 & 5 & 28. & $\mathrm{p} 28$ & 109 & 27 & 2 \\
\hline 14. & P14 & 49 & 1 & 2 & 29. & $\mathrm{p} 29$ & 106 & 2 & 1 \\
\hline 15. & P15 & 69 & 39 & 2 & 30. & $\mathrm{p} 30$ & 125 & 1 & 1 \\
\hline
\end{tabular}

(Type 1.Arch, 2.Left loop 3.Right loop, 4.Whorl, 5.Tented arch)

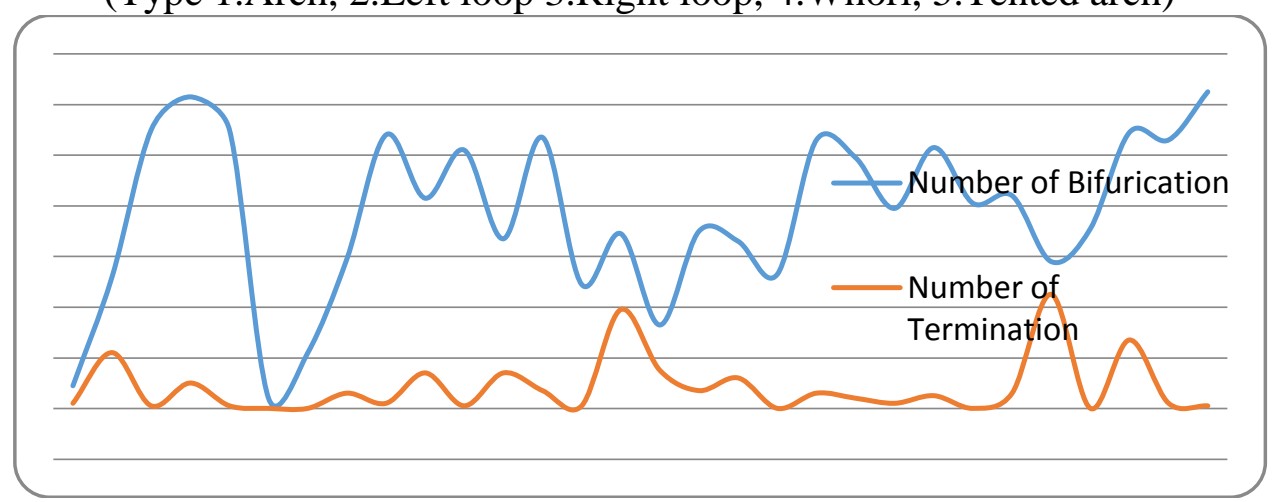

Fig 4.8 Termination and Bifurication of dataset 
Fig 4.8 representes Termination and Bifurication orientation values for finger print images and these values are find from minutiae extraction and region of interest. We used these values to find the typres of finger print. We achieved 93.3.\% correct classification of finger print is shown in Table 2.

Table 2. Accuracy of Finger print Identification

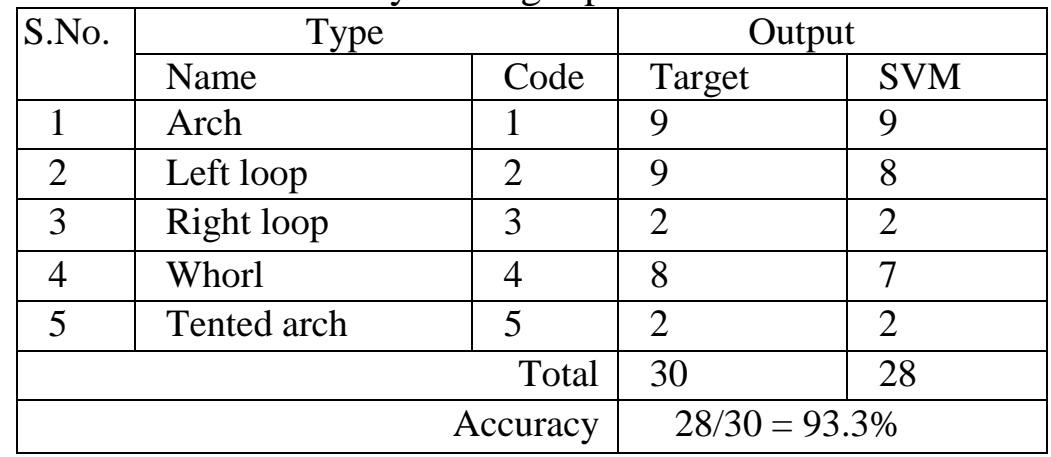

Fingerprint Classification using SVM

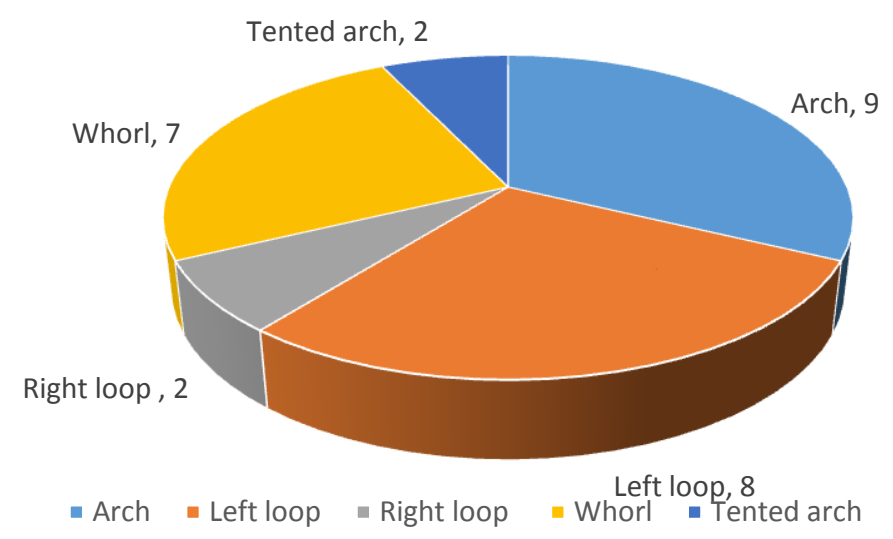

Fig 4.9 Finger Print types Classification

Fig 4.9 represents thirty datasets trained for minutiae extraction using SVM algorithm. The number of termination orientation values for applied for false minutiae removal and applied for region of interest then create the mask and suppress the minutiae values. Twenty eight datasets in the table were properly classified the finger print types and two datasets were not suitable for this algorithm.

\section{CONCLUSION}

This paper discussed about the classification finger print images. The above implementation was an effort to understand how Fingerprint identification is used as a form of biometric to recognize identities of human beings. It includes all the stages for minutiae extraction from fingerprints. Various standard techniques are used in the intermediate stages of processing. Fingerprints are classified into five classes (arch, tented arch, left loop, right loop and whorl). The singular points are quite frequently features for classification. The taken work has reached its success in accuracy. Almost it provides the expected result. About 93 per cent precision has been achieved, using the SVM algorithm. This work will help in the authentication of aadhaar card and this result offers hope for the more research works related to this subject. Future research work can be carried out to improve the quality of the image by improving the image enhancement technique and develop a better matching technique. 
DOI: https://dx.doi.org/10.26808/rs.ca.i8v1.20 International Journal of Computer Application (2250-1797)

Issue 8 Volume 1, January- February 2018

\section{REFERENCES}

I. S. Pankanti, S. Prabhakar, and A. K. Jain, "On the Individuality of Fingerprints." IEEE Trans. PAMI, Vol. 24, page no. 1010-1025, 2002.

II. Anil k. Jain, UmutUludag, "Hiding Biomeric Data", IEEE transactions on Pattern Analysis and Machine Intelligence, vol. 25, no. 11, pp. 1494 - 1498, November 2003.

III. A.K. Jain, R. Bolle, S. Pankanti (Eds.), "Biometrics: Personal Identification in Networked Society", Kluwer Academic Publishers, Dordrecht, 1999.

IV. Anil K. Jain and David Maltoni., "Handbook of Fingerprint Recognition." Springer Verlag New York, Inc., Secaucus, NJ, USA, 2003.

V. A.R. Roddy and J.D. Stosz, "Fingerprint features-statistical analysis and system performance estimates" Proc. IEEE, vol. 85, no. 9, page no. 1390-1421, 1997.

VI. Luk, S.H. Leung, "A Two Level Classifier For Fingerprint Recognition.” in Proc. IEEE 1991 International Symposium on CAS, Singapore, page no. 2625-2628, 1991.

VII. Gour, T. K. Bandopadhyaya and R. Patel, "ART and Modular Neural Network Architecture for Multilevel Categorization and Recognition of Fingerprints," Third International Conference on Knowledge Discovery and data Mining, pp. 536-539, 2010.

VIII. Yong-xia Liu, Jin Qi and RuiXie, "A new detection method of Singular Points of finerprints based on neural network," 3rd IEEE International conference on Computer Science and Information Technology, vol. 01, pp. 301-305, 2010.

IX. J.D. Stosz and L.A. Alyea, "Automated system for fingerprint authentication using pores and ridge structure." Proc. of the SPIE Automatic Systems for the Identification and Inspection of Humans, Volume 2277, page no. 210-223, 1994.

X. P. Melin, D. Bravo and O. Castillo, "Fingerprint recognition using modular neural networks and fuzzy integrals for response integration," IEEE International Joint Conference on Neural Networks, vol. 04, pp. 2589-2594, 2005.

XI. Moheb R. Girgis, Tarek M. Mahmoud, and TarekAbd-El-Hafeez, "An Approach to Image Extraction and Accurate Skin Detection from Web Pages." World academy of Science, Engineering and Technology, page no. 27, 2007.

XII. Manvjeet Kaur, Mukhwinder Singh, AkshayGirdhar, and Parvinder S. Sandhu, "Fingerprint Verificationm using Minutiae Extraction Technique." World academy of Science, Engineering and Technology, page no. 46, 2008.

XIII. N. K. Ratha, K. Karu, S. Chen, and A. K. Jain, "A real-time matching system for large fingerprint databases." Transactions on Pattern Analysis and Machine Intelligence, 18(8): page no. 799-813, 1996.

XIV. MayankVatsa, Richa Singh, AfzelNoore, Sanjay K. Singh, "Combining pores and ridges with minutiae for improved fingerprint verification." Elsevier, Signal Processing 89, page no. 2676-2685, 2009.

XV. K. Nishimura, S. Kishida, and T. Watanable, "Improvement of preprocessing method on fingerprint identification system by layered neural networks," IEEEEurasip Nonlinear Signal and Image Processing, 2005

XVI. S. Sato and T. Umezaki1, "A fingerprint segmentation method using a recurrent neural network," 12th IEEE Workshop on Neural networks for signal processing, pp. 345-354, 2002.

XVII. Qijun Zhao, Lei Zhang, David Zhang, Nan Luo, “Adaptive Pore Model for Fingerprint Pore Extraction." IEEE, 978-1-4244-2175, 2008.

XVIII. J. S. Bartunek, M. Nilsson, J. Nordberg and I.Claesson, "Neural Network based Minutiae Extraction from Skeletonized Fingerprints," IEEE Region 10 Conference on TENCON, pp. 1-4, 2006. 
DOI: https://dx.doi.org/10.26808/rs.ca.i8v1.20 International Journal of Computer Application (2250-1797) Issue 8 Volume 1, January- February 2018

XIX. Marius Tico, Pauli Kuosmanen, "Fingerprint Matching using an Orientation based Minutia Descriptor", IEEE transactions on Pattern Analysis and Machine Intelligence, vol. 25, no. 8, pp. 1004-1014, August 2003.

XX. L. Hong, Y. Wan and A. Jain,"Fingerprint Image Enhancement: Algorithm and Performance Evaluation",

XXI. IEEE tranactions on Pattern Analysis and Machine Intelligence, vol. 20, no. 8, pp. 777 789, August 1998.

XXII. M. Kamijo, "Classifying fingerprint images using neural network: deriving the classification rate," IEEE International Conference on Neural Networks, vol. 03, pp. 1932-1937, 1993.

XXIII. S. Karungaru, K.Fukuda , M.Fukumi and N.Akamatsu, "Classification of fingerprint images into individual classes using Neural Networks," $34^{\text {th }}$ Annual Conference on Industrial Electronics, pp. 1857-1862, 2008. 